\title{
Filigrane
}

Écoutes psychanalytiques

\section{Réflexions sur la construction des interdits}

\section{Maurice Berger}

Volume 26, numéro 2, 2017

La terreur des enfants : deuxième partie

URI : https://id.erudit.org/iderudit/1055357ar

DOI : https://doi.org/10.7202/1055357ar

Aller au sommaire du numéro

Éditeur(s)

Revue Santé mentale au Québec

ISSN

1192-1412 (imprimé)

1911-4656 (numérique)

Découvrir la revue

\section{Citer cet article}

Berger, M. (2017). Réflexions sur la construction des interdits. Filigrane, 26(2), 9-26. https://doi.org/10.7202/1055357ar

\section{Résumé de l'article}

Dans le contexte d'une crise générale de l'autorité tant aux niveaux politique que social et familial, il y a lieu de questionner la fonction psychique de l'autorité et des interdits dans le développement psychoaffectif de l'enfant et de l'adolescent. Pour s'assurer que l'autorité ne bascule pas en autoritarisme ou en simple arbitraire, il faut néanmoins clarifier au préalable la distinction entre interdit organisateur et interdit désorganisateur. Le rôle de l'interdit est, entre autres, de permettre de lier entre eux les désirs pulsionnels avides, violents et sexuels, la crainte de perdre l'amour d'autrui et la rencontre avec la réalité. Nous examinerons en ce sens la construction des interdits au niveau intrapsychique, social et de la loi, et ce, de l'enfance à l'âge adulte, en soulignant les conflits qui peuvent survenir à chaque étape de ce développement. Nous serons ainsi à même d'énumérer quelques difficultés familiales typiques ayant trait aux interdits ainsi que les façons de les aborder en clinique.
Ce document est protégé par la loi sur le droit d'auteur. L'utilisation des services d’Érudit (y compris la reproduction) est assujettie à sa politique d'utilisation que vous pouvez consulter en ligne.

https://apropos.erudit.org/fr/usagers/politique-dutilisation/ 


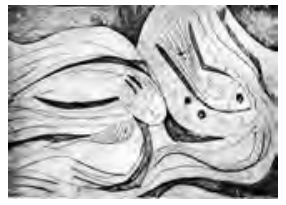

\title{
Réflexions sur la construction des interdits
}

\author{
Maurice Berger
}

RÉSUMÉ: Dans le contexte d'une crise générale de l'autorité tant aux niveaux politique que social et familial, il y a lieu de questionner la fonction psychique de l'autorité et des interdits dans le développement psychoaffectif de l'enfant et de l'adolescent. Pour s'assurer que l'autorité ne bascule pas en autoritarisme ou en simple arbitraire, il faut néanmoins clarifier au préalable la distinction entre interdit organisateur et interdit désorganisateur. Le rôle de l'interdit est, entre autres, de permettre de lier entre eux les désirs pulsionnels avides, violents et sexuels, la crainte de perdre l'amour d'autrui et la rencontre avec la réalité. Nous examinerons en ce sens la construction des interdits au niveau intrapsychique, social et de la loi, et ce, de l'enfance à l'âge adulte, en soulignant les conflits qui peuvent survenir à chaque étape de ce développement. Nous serons ainsi à même d'énumérer quelques difficultés familiales typiques ayant trait aux interdits ainsi que les façons de les aborder en clinique.

Mots clés: Interdit, autorité parentale, limites, incestuel.

\begin{abstract}
In the context of a general crisis of authority at the political, social and familial levels, it seems all the more crucial to question the psychic function of authority in the psycho-affective development of children and adolescents. To ensure that authority does not turn into authoritarianism and arbitrary power, it is necessary to clarify beforehand the distinction between organizational and disorganizational interdicts. The positive role of the interdict is to link avid, violent and sexual drive desires with the fear of losing the love of others and the encounter with reality. In this regard, we will examine the construction of the intrapsychic, social and legal prohibitions, from childhood to adulthood, highlighting the conflicts that can arise at each stage of this development. We will then take a look at some typical difficulties that parents live relating to authority and limits and present some ways to address them in clinic.
\end{abstract}

Keywords: Interdict, parental authority, limits, incestual.

\section{Introduction}

ui aime parler des interdits? Pas grand monde. Mais peut-on éviter une réflexion sur ce sujet alors que cette question est de plus en plus présente dans la pratique clinique, ce qu'on peut mettre en lien avec la crise actuelle de l'autorité dans le champ social? Quelques exemples montrent 
l'intensité de ce problème en France, pays dévasté par des raisonnements idéologiques.

- Le slogan de Mai 68: «il est interdit d'interdire».

- Il y a un mois, une manifestation nationale à Saint-Étienne, ville où j'habite et où l'on fabrique certaines armes des policiers, sur le thème: «Il faut désarmer la police», manifestation violente qui a fait des dizaines de milliers d'euros de dégâts.

- Des policiers brûlés vifs dans leur voiture en feu, violences gravissimes effectuées pas seulement par des dealers gênés dans leur trafic par la présence des forces de l'ordre, mais aussi par des individus appartenant à des groupes anarchistes, ce qui rappelle l'époque d'Action directe, ou des Brigades rouges en Italie.

- Une augmentation importante chaque année du nombre (environ 20 par jour) de policiers blessés et tués pendant l'exercice de leurs fonctions.

- Un droit implicite de tabassage sans emprisonnement s'il ne s'agit pas d'une récidive, et même si la victime a perdu connaissance et a des lésions corporelles importantes à la suite d'une agression.

- La Cour de cassation qui juge illégale l'expulsion des occupants d'un terrain occupé illégalement.

- Un agriculteur poursuivi pour avoir tapé avec sa pelleteuse sur la voiture d'individus volant son matériel. Il n'avait que ce moyen pour les empêcher de s'enfuir avec ses biens. Les voleurs ont été condamnés à 450 euros d'amende et l'agriculteur à 2500 euros d'amende pour destruction de leur outil de travail.

- Une ministre de la Justice qui considère que tous les délinquants sont d'abord des victimes et qu'il ne faut donc pas les mettre en prison, soutenue par le Syndicat de la magistrature, syndicat d'extrême gauche auquel adhèrent $24 \%$ des magistrats syndiqués, et qui, à sa création, avait comme but d'abolir totalement la prison.

- Etc., tous les jours.

Il est très angoissant de vivre dans un tel monde. C'est l'autorité qui permet d'énoncer les interdits, et son dysfonctionnement crée du malêtre, alors que l'autorité de bon aloi, décrite plus loin, crée du bien-être. Pour autant, l'exercice de l'autorité n'est pas une donnée en soi, c'est un processus qui n'est jamais «réglé» une bonne fois pour toutes en chacun de nous.

Quelques autres remarques générales. Tout d'abord, deux citations. 
Lacordaire: «Entre le fort et le faible, entre le riche et le pauvre, entre le maître et le serviteur, c'est la liberté qui opprime et la loi qui affranchit». Il est intéressant de mettre cette phrase en lien avec ce que souligne Philippe Jeammet, psychiatre psychanalyste spécialiste de l'adolescence: «L'évolution sociale actuelle avec son questionnement des limites et la perte d'un consensus social suffisamment fort concernant les règles éducatives entrent en résonance avec la dynamique de l'adolescence, en redoublant ses effets. Les plus vulnérables de ces adolescents sans limites contenantes suffisantes sont abandonnés à leurs contraintes émotionnelles et à leurs passages à l'acte». Je commente: la tyrannie des pulsions peut être plus forte que la supposée tyrannie des interdits. Nous commençons à percevoir l'articulation entre le fonctionnement sociétal et le fonctionnement intrapsychique.

Et Kant: «Les parents n'ont à l'ordinaire d'autre objet de soin que la réussite de leurs enfants dans le monde. Or ils devraient les éduquer pour que naisse un meilleur état futur».

Du côté de la loi, le texte légal consacré à l'autorité parentale en France, le Code civil, débute par l'Article 371: «L'enfant doit honneur et respect à ses père et mère ${ }^{1}$.» Peu de professionnels et d'adultes en général s'appuient sur cette phrase qui a pourtant une fonction tierce intéressante. Elle montre que les conflits du moment ne sont pas simplement intrafamiliaux. Ils s'inscrivent dans ce que la société et le législateur ont défini comme condition indispensable à la vie sociale du pays, à savoir une asymétrie entre adultes géniteurs et enfant.

À ces multiples axes concernant la construction des interdits, on peut ajouter les champs culturels et ethnologiques: ainsi dans notre culture, une femme peut se promener dans la rue les cheveux découverts et avec un chemisier décolleté, alors que ceci est interdit dans d'autres cultures parce que toute partie visible de sa peau est considérée comme excitant le désir masculin donc indécente.

Comme l'a indiqué Carel (2002), nous nous questionnons en permanence à titre individuel et en tant que citoyens sur la limite entre autorité et autoritarisme. Et il faut souligner dès maintenant un processus de retournement fréquemment à l'œuvre pour éviter d'être confronté à ce que la pose d'un interdit déclenche comme malaise: Lacroix indique que lorsque dans notre pratique institutionnelle nous posons un interdit, immédiatement se produit un mouvement de bascule, et l'agresseur, quels qu'aient été les actes qu'il a commis sur autrui, devient une victime aux yeux des autres professionnels et de soi-même (1999). 
Pour compliquer ce tableau, on constate que lorsqu'un interdit a été levé, aboli par une jurisprudence quelconque ou par une évolution des mœurs, il est difficile de revenir en arrière. Toute tentative de rétablissement ou d'établissement d'un interdit même structurant et nécessaire est qualifiée de «retour à l'ordre moral», «réactionnaire», voire fréquemment en France de «fasciste». Et les médias, qui vont souvent dans le sens de la séduction, jouent un rôle majeur dans la déliaison, en tournant systématiquement ces tentatives en dérision. La dérision, perversion de l'humour, et son intention destructrice sont un mode de fonctionnement parmi les plus immédiatement disponibles au psychisme humain et les plus économiques à court terme: mieux vaut détruire ce qui est imparfait et laborieux chez l'autre, et ainsi garder l'illusion de solutions idéales obtenues sans effort. Or le rôle de l'interdit est, entre autres, de permettre de lier entre eux les désirs pulsionnels avides, violents, sexuels émanant du Ça, la crainte de perdre l'amour d'autrui, la rencontre avec la réalité. Ce travail du Moi, jamais terminé, est toujours imparfait et il est donc facile de s'en moquer.

Pour en terminer avec ces généralités, ajoutons que l'interdit, ou le fait de poser des limites, introduit une différence entre le sujet qui l'émet et le destinataire: interdit de faire telle chose, interdit de pénétrer dans tel territoire, qui peut être le territoire national, ou la chambre des parents à certains moments, etc. D’une manière générale, la valeur d'une pensée se définit, entre autres, par la capacité de penser la limite en général, et les limites nécessaires dans toute vie groupale en démocratie. Et cette différence implique une asymétrie entre les sujets, éventuellement une exclusion, donc une certaine violence.

\section{Interdit organisateur et désorganisateur}

Oui, l'interdit est porteur d'une violence, mais il faut distinguer la violence organisatrice, qui permet à des enfants et à des adultes d'organiser leur vie psychique et de réguler la vie sociale, et la violence désorganisatrice qui livre le sujet au monde pulsionnel d'autrui, à ses propres pulsions, et qui est incompatible avec une vie groupale où règne un minimum de justice et de protection corporelle. Car la loi a d'abord pour but de protéger le plus faible du plus fort.

L'interdit organisateur ne dépasse pas le niveau minimum de violence nécessaire; il n'est pas malveillant; il s'accompagne de paroles de la part de l'adulte interdicteur pour donner à l'enfant de bonnes raisons de se soumettre; il ne repose pas sur l'arbitraire du parent, il est justifié sans que 
l'adulte ait forcément à le prouver malgré la liste interminable des «pourquoi? »; après avoir énoncé l'interdit, le parent laisse un délai à l'enfant pour lui donner la possibilité de se contenir, de faire siennes les raisons proposées par l'adulte. Surtout, l'interdit peut être accepté parce qu'en arrièreplan existe un amour inconditionnel réciproque entre l'enfant et le parent, et parce qu'existe une relation d'amour tendre. Dans l'éducation, l'interdit nécessite un fond relationnel de tendresse.

Poser un interdit nécessite donc d'avoir le courage de se différencier d'autrui, d'affronter un refus. Le courage n'existe pas en soi, il consiste à avoir peur et à faire quand même. Poser un interdit signifie accepter de ne pas être aimé temporairement, ce qui nécessite d'avoir un narcissisme solide mais non excessif, et de ne pas craindre de se sentir seul - car on est souvent solitaire dans ces circonstances. On comprend donc qu'il soit toujours plus facile de dire oui, c'est pour cela que l'inverse de l'autorité, c'est la séduction, plaire, chercher à se faire aimer.

\section{Comment se construisent les interdits}

La construction des interdits se situe à trois niveaux: intrapsychique, sociétal, judiciaire.

1) Au premier niveau, intrapsychique, un parent transmet à son enfant des interdits qui, souvent, lui ont été transmis par ses propres parents et dont il a ressenti le bien-fondé.

Ces interdits ont un sens différent selon les processus en jeu chez l'enfant, depuis son plus jeune âge jusqu'à l'adolescence, et mobilisent chez l'adulte des niveaux d'identification, d'inquiétude et de culpabilité différents. Le risque existe qu'un interdit (ou une absence d'interdit) adapté pour un âge soit énoncé à une période de la vie où il ne convient pas.

a) Pendant la première année de la vie

Winnicott (1963) souligne que le début de la fonction organisatrice des parents se situe dès les premiers mois de la vie: la sollicitude, c'est-à-dire le fait qu'un individu se sent concerné et responsable de ce qui arrive à autrui (au sens de la culpabilité), ne peut apparaître «que si le nourrisson a disposé d'une "mère-environnement" de bonne qualité, une personne veillant à écarter l'imprévisible et qui, d'une façon active, soigne et dirige». Donc un fond relationnel stable, prévisible, et un amour inconditionnel, la préoccupation maternelle primaire, sont nécessaires. Et sur ce fond, diverses règles organisent 
la vie pulsionnelle du bébé. Pas d'organisation sans règles, et les règles sont désorganisatrices si elles n'ont pas un fond relationnel adéquat. Il faut ainsi articuler être tenu et être contenu, deux facettes différentes du holding. On comprend qu'ultérieurement, dans les soins, l'éducation, la rencontre avec la justice, etc., ne pas être contenu puisse être vécu comme un lâchage, un noninvestissement. Contenir renvoie à trois notions: 1) Procurer une enveloppe, et ceci concerne en particulier la corégulation des émotions, dont on sait que la période critique cérébrale qui permet son acquisition se situe entre 8 et 24 mois; 2) Indiquer une limite, une bordure, une ligne de démarcation entre deux territoires, et qui peut avoir une dimension subjective; ceci concerne les moments où l'adulte détourne certaines attaques lors de la période de destructivité, vers 4-5 mois; 3) L'inter-dit, qui consiste à défendre, souvent en référence à une contrainte sociale ou législative.

b) L'apparition $\mathrm{du}$ «non»

Ces interdits se manifestent plus clairement lorsqu'apparaît le «non» de l'enfant vers 12-15 mois, dont Spitz a indiqué que c'était le premier mot « concept», car l'enfant le généralise rapidement à un ensemble de situations.

Le «non» de l'enfant est alors une réponse au «non» de l'adulte (Carel, 2002). Ce «non» de l'enfant questionne la possibilité du parent de réaliser le deuil originaire. Racamier y voit ainsi

un processus psychique fondamental par lequel le Moi, dès ses prémices et jusqu'à la mort, renonce à la possession totale de l'objet, fait son deuil d'un unisson narcissique absolu, et par ce deuil même, qui fonde ses origines, opère la découverte ou l'invention de l'objet. Le Moi établit donc ses origines en reconnaissant qu'il n'est pas le maître absolu de ses origines.

Dit plus simplement, c'est un mouvement de différenciation initié par l'adulte qui introduit la perte de l'illusion de pouvoir être tout l'un pour l'autre. Cela parait théorique, mais dans la réalité, c'est un événement qui peut être bouleversant par la violence affective qu'il implique, déprimant de part et d'autre de par la perte qui en découle. N'oublions pas, et Freud l'a souligné le premier, à quel point nos enfants sont nos prolongements narcissiques; d'ailleurs ce sont le plus souvent les enfants des autres qu'on trouve mal élevés, pas les siens. De plus, ce «non» nécessite que le parent ne se représente pas son enfant petit comme détruit par l'interdit, c'est-à-dire ayant perdu l'amour parental, donc abandonné. 
L'interdit du parent fait déchoir sa majesté le bébé. Lorsque le parent émet ce non à cette période de la relation qu'il a avec son enfant, il se fait réellement violence, d'autant plus que l'enfant lutte contre cette différenciation, veut garder sa place exclusive, son pouvoir sur l'adulte. Qui, normalement constitué, aime le conflit? L'interdit organisateur consiste à accepter le conflit, non pas pour être le plus fort, mais pour pouvoir vivre ensemble, et il repose sur l'expérience que l'adulte a de la vie et sur sa représentation du futur, afin de faire un enfant capable de vivre dans le groupe social, un citoyen, comme Kant l'a indiqué, et pas seulement destiné à vivre dans le groupe familial.

À cette période de la vie, ne parle-t-on pas trop facilement du rôle de tiers du père ou, ce qui est un peu mieux, de la fonction paternelle. Tout d'abord, un certain nombre de pères, qui n'ont aucune envie de réaliser un travail de deuil originaire, embrassent leur enfant sur la bouche sans que cela leur pose le moindre problème, dans une sorte de relation en miroir absolu en plus d'un mélange de place plus ou moins sexualisé. Et parler à ces pères de la différence entre les bisous entre parent et enfant et les baisers entre amoureux amène une réponse de surface: s'ils cessent souvent ce genre de contact corporel devant nous, ont-ils réellement pris conscience de l'inadéquation de leur attitude? Mais à ce moment, l'enfant, lui, est attentif à notre parole.

Par ailleurs, comme l'indique Albert Ciccone (2016), le rôle du père, ce n'est pas d'interdire, ou en tout cas, cela ne se résume pas à ça. Sinon, il serait peu intéressant d'être père. Il paraît plus adapté de parler de «fonction adulte» qui devrait être mise en œuvre chez tout parent, qu'il soit père ou mère. Et cette fonction se situe à plusieurs niveaux: l'interdit surmoïque que propose l'adulte est alors un Surmoi bienveillant, et non malveillant, anti-croissance du Moi. Carel (2016) parle d'«épreuve du non surmontée ensemble», car il s'agit aussi d'une épreuve pour le parent. Il faut rappeler que le Surmoi est trop souvent réduit à sa fonction interdictrice; il est aussi régulateur, au sens de la nécessité de l'autoconservation du sujet («ce ne serait pas bon pour toi que je te laisse manger tout le paquet de bonbons»), et consolateur («moi aussi à ton âge, j'avais envie de...»).

De plus, en expliquant pourquoi il interdit, le parent prescrit des valeurs, des idéaux, il construit une offre surmoïque: le Surmoi est proche du Moi idéal, modèle auquel l'enfant a envie de ressembler. Une de ces valeurs peut consister à s'identifier à ce que ressent l'autre, à se mettre à sa place, à penser qu'il peut être gêné quand on fait du bruit, quand on passe devant lui à un 
guichet. «Si tout le monde faisait comme moi, qu'est-ce que cela donnerait... : le Surmoi a donc une organisation spatiale.

\section{c) L'organisation anale}

À un tour de spire supérieur, au moment de l'organisation anale des pulsions, le «non» de l'enfant se situe dans un autre registre. Jusqu'alors, l'enfant était sous la tyrannie de l'objet, au sens où, dans l'état d'impuissance où il se trouvait, la satisfaction de ses désirs dépendait totalement de ses parents. Maintenant, il a acquis la marche, ses mains sont libres donc il peut attraper les objets et éventuellement frapper; il peut demander, exiger, même si ses capacités d'expression sont un peu décalées par rapport à ses capacités de compréhension; et comme l'indiquent Grunberger (2003) et Roussillon (2014), la dépendance se retourne en indépendance absolue, le sujet veut faire subir à l'autre la dépendance qu'il a subie jusqu'alors. La relation est donc avant tout énergétique entre le sujet et son environnement; il en va d'un rapport de force qui fait passer au second plan les valeurs liées au contenu. L'idéal de l'enfant est alors une relation maître-esclave. Pour conquérir son autonomie, il se place au-dessus du parent, c'est la période des caprices. L'idéal, c'est de faire tout seul, d'être dans une position de maîtrise, c'est pour cela que l'organe de l'analité, c'est le muscle, dont l'exemple est le sphincter anal qui permet de décider quand et où l'on veut se séparer de ses matières fécales qui sont à l'intérieur de son corps donc inaccessibles aux autres.

Cette nouvelle épreuve pour enfant et parent ne peut se résoudre que par la négociation, tous les désirs ne peuvent pas être satisfaits au même moment, tout de suite: il faut tenir compte des souhaits de chacun, et le parent joue alors un rôle de «chef d'orchestre» entre les différents désirs des membres du groupe familial. Mais ceci peut être insuffisant, et apparaît alors la nécessité d'une butée à la tyrannie de l'enfant, sous diverses formes: l'envoyer dans sa chambre, le rabrouer d'une voix forte, voire - si l'enfant, malgré ces mesures, nargue l'adulte de manière importante et tente ainsi d'abolir la différence entre lui et l'adulte - une fessée bien tempérée.

Afin d'éviter cette deuxième épreuve du non, le parent peut trouver une solution économique à court terme, mais pas pour la suite. Elle consiste à émettre un interdit qui s'accompagne de messages où le parent s'auto-disqualifie, par son ton de voix, parfois suppliant, qui montre qu'il s'excuse en même temps. Ou encore, le parent émet un interdit tellement énorme que l'enfant perçoit tout de suite qu'il ne sera pas tenable. Enfin, le parent peut 
demander à l'environnement extérieur de tenir ce rôle. Je marche et, devant moi, sur le trottoir, un enfant de cinq ans refuse d'obéir à sa mère. Cette dernière lui dit: «si tu n'obéis pas, le monsieur (c'est moi) va te gronder». Dans cette option, certains parents se défaussent en demandant aux corps sociaux (école, club sportif, etc.) de réguler la non-obéissance, l'impertinence et la violence de leur enfant, ce dont les professionnels se plaignent, car cela les détourne de leurs fonctions. Les parents attendent aussi que les pairs de leur enfant tiennent ce rôle lors de la confrontation de leur rejeton avec le groupe des autres enfants de son âge, avec un risque majeur: que dans ce groupe règne la loi du plus fort.

C'est à cette période de la vie que poser un interdit à son enfant signifie le plus qu'on accepte de ne pas être aimé par lui pendant un moment, voire d'être détesté. «Je ne t'aime plus » crie l'enfant à son parent, et parfois même «je voudrais que tu sois mort». On constate l'asymétrie nécessaire entre enfant et parent: un parent peut normalement supporter de ne pas être aimé temporairement par son enfant (c'est plus simple à dire qu'à tolérer dans les faits), alors que l'amour de ses parents est indispensable pour l'enfant, qui ne peut pas se permettre de s'imaginer ne pas être aimé par eux.

Mais revenons sur «je ne t'aime plus». L'enfant peut se permettre de dire cette phrase parce qu'il sait qu'elle n'est qu'une pensée et ne correspond pas à la réalité; sauf si le parent a gardé un mode de pensée magique dans lequel le mot, la pensée, le fantasme, est l'équivalent de l'acte. Pour une partie de son fonctionnement psychique, un tel adulte n'a pas accès à la transitionnalité. Le problème est alors que l'interdit porte sur la pensée et non pas sur l'acte: «Je t'interdis de penser ça». Rappelons qu'il existe plusieurs niveaux d'interdits : celui de faire certains actes, celui de dire certaines choses dans l'espace public, comme l'incitation au racisme, etc. Mais dans l'espace privé, on peut dire certaines choses sans que cela signifie qu'on souhaite leur réalisation. Enfin existe un domaine où il n'y a pas d'interdit, c'est celui de la pensée «en soi»: on a le droit de tout penser, même les choses les plus horribles et les plus perverses.

Ainsi Fabien B, sept ans, dit un jour à sa mère qu' il a envie de «zigouiller » son petit frère âgé de dix-huit mois. Les parents prennent cela pour argent comptant et expliquent à Fabien que lorsqu'il est né, son frère aîné n'avait pas été très content non plus, et qu'ils se sont occupés de lui, Fabien, comme ils s'occupent du bébé maintenant. Il n'a donc pas à être jaloux et à vouloir sa mort. Interaction banale; mais, si on approfondit un peu, on découvre un soubassement plus inquiétant. Un peu plus tard, Mme B., effrayée, déclare 
que Fabien ment. Elle l'a fait jurer qu'il disait la vérité, sur la tête de ses grands-mères qui sont mortes, et il n'a pas hésité à jurer, alors qu'il mentait. Je demande quelques explications et Mme B. me précise que les grandsmères ont dû effectivement entendre que Fabien disait un mensonge. Elles représentent donc un Surmoi qui voit et entend tout et peut deviner les mensonges. Mme B. précise alors qu'elle-même ne pourrait jamais aller dans un cimetière la nuit: elle aurait peur que les morts ne l'attaquent, car ils ne la reconnaîtraient pas. On constate, dans ce monde de pensée magique, à quel point la différence entre fantasme et réalité se révèle fragile.

Cet exemple montre les croyances parentales intouchables qui peuvent être en jeu derrière ce qui semble être un problème éducatif relativement banal, qu'on aurait simplement envie d' "assouplir»-j'y reviendrai.

Évoquons rapidement ici une autre dimension de l'interdit à cette période et qui persiste même ultérieurement: prosaïquement, pour énoncer un interdit d'une manière qui aide l'enfant à structurer sa vie pulsionnelle, l'adulte doit accepter d'être délogé de sa tranquillité narcissique pour s'opposer à son enfant, c'est-à-dire arrêter temporairement d'être avachi devant la télévision ou incrusté face à son jeu vidéo.

d) La période œdipienne.

À la période odipienne, les interdits prennent une autre tournure: ils ne sont pas énoncés tels quels mais sous la forme de déplacements et d'exclusion. Ce qui les spécifie est le refoulement par le parent de la dimension sexualisée de ses propres pensées face à certaines paroles et attitudes sexualisées de son enfant. Ce refoulement est une des caractéristiques d'une fonction parentale adéquate. Mais ce refoulement ne signifie pas déni, alors qu'on constate comment certains parents en consultation innocentent trop facilement la manière très sexualisée dont l'enfant colle leur corps contre eux: tel garçon par exemple tripote les seins de sa mère, une fillette s'empare de l'index de son père en regardant sa mère de manière triomphante et en disant «ça, c'est à moi », tout en étant couchée à plat ventre sur le père qui s'est allongé sur mon divan et qui ne voit là qu'un jeu de tout-petit. Là encore, la question est d'oser blesser l'enfant narcissiquement en lui indiquant qu'il n'est «qu'un enfant».

Au garçon qui déclare à sa mère «Quand papa sera mort, je me marierai avec toi», paroles qui existent réellement, le parent propose des déplacements: il ne dit évidemment pas «tu ne coucheras pas avec ta mère», ce qui introduirait du sexuel, mais «ce n'est pas possible qu'un enfant se marie 
avec un parent» - c'est l'énoncé de l'interdit -, puis «quand tu seras grand, tu pourras te marier avec ta femme à toi ». Il y a déplacement d'objet - une autre personne du sexe féminin - et déplacement dans le temps - «quand tu seras plus grand...». Mais il ne faut pas se leurrer, l'enfant qui constate que sa tentative a échoué ne renonce qu'en surface, et le désir reste bien ancré en lui. Ainsi le fils d'une collègue psychologue lui annonce qu'il veut se marier avec sa sœur cadette. Sa mère lui explique qu'un frère ne peut pas se marier avec sa sœur. Il réfléchit un peu, puis ajoute: «Ça ne fait rien, je me marierai quand même avec elle».

L'interdit prend aussi une autre forme: l'exclusion des moments réservés au plaisir du couple. Il est indiqué à l'enfant qu'il ne peut pas aller voir certains films avec ses parents parce qu'ils sont pour les grands, aller chez certains amis car cela se passe tard le soir, etc. Ainsi, lorsqu'ils vont voir un film pour adultes, des parents incapables de se positionner comme couple face à leurs enfants leur expliquent qu'ils vont à une conférence sur le cinéma, activité intellectuelle peu attirante. Ils pensent ainsi éviter de les mettre dans une situation d'envie qui serait, d'après eux, intolérable. Les conséquences sont plus importantes qu'on peut l'imaginer: l'enfant ne peut pas fantasmer sur le plaisir qu'ont les adultes entre eux, et c'est la construction des fantasmes originaires qui est en péril; qu'est-ce qui fait que deux adultes se séduisent, quelle est l'origine de la différence des sexes, comment se déroule la conception? Et il peut en découler une position de collage infantile au couple ou d'autres troubles.

D’une manière générale, les difficultés à poser des interdits décrites cidessus peuvent paraître bénignes. En réalité, elles sont souvent très ancrées chez les parents et difficilement mobilisables. Elles peuvent renvoyer à des angoisses d'abandon ou de catastrophe massivement projetées sur l'enfant, considéré comme beaucoup plus fragile en soi et face à l'interdit qu'il l'est réellement. Les interdits excessifs peuvent aussi parfois renvoyer à des angoisses de perte de contrôle. Derrière le bon niveau socio-culturel de certains parents, ces difficultés qui ne sont pas de l'ordre de l'éducatif sont proches d'un registre très archaïque.

e) L'adolescence

À l'adolescence, les parents risquent de constater l'inefficacité de leurs interdits. L'affirmation de soi prend la forme d'un désir de transgression, d'un besoin de savoir ce qu'il y a derrière les règles, les interdits des adultes, maintenant que le sujet a l'autonomie, la force, et les capacités sexuelles des 
grands. À cela s'ajoute le sentiment d'impunité lié à l'«anonymat» de la transgression en groupe. Étant donné leur surdité aux interdits autoconservateurs des adultes, certains adolescents ne trouvent la limite qu'à l'extérieur d'eux. Cette butée peut prendre la forme d'une rencontre traumatique de leur corps avec un obstacle, c'est le problème des conduites à risque, ou la forme de la rencontre avec la loi, dont il peut être souhaitable qu'elle ait lieu le plus rapidement possible de façon matérialisée.

2) Le deuxième niveau de construction des interdits se situe au niveau social, il est constitué par les messages venant du groupe dans lequel on vit. Le groupe social exerce une influence qui prend la forme du «qu'en dira-t-on?», «tu me fais honte», «ça ne se fait pas», ce que Freud nomme l'opinion publique. Cette honte groupale est structurante à la différence de la honte désorganisatrice qui touche l'identité, l'origine, l'essence même du sujet: «sale juif», «sale arabe», «sale homosexuel», etc. On constate que pour tenir leurs positions éducatives, beaucoup de parents s'étayaient autrefois sur la crainte du regard d'autrui, du «qu'en dira-t-on?». La honte de présenter un enfant mal élevé au groupe extérieur à la famille nucléaire constituait pour eux un appui essentiel. Or notre fonctionnement groupal en France n'est plus suffisamment porteur d'interdits. La société est devenue séductrice, excitante, au lieu d'être d'abord contenante.

On comprend que poser un interdit dans ce contexte nécessite de la part de l'adulte la capacité d'affirmer qu'il pense différemment, non seulement de l'enfant, mais des autres adultes, capacité de se différencier de ce que Bion nomme la «mentalité de groupe» (1959): «Tout le monde fait comme ça maintenant...» Combien de citoyens sont capables de se tenir debout face au groupe? C'est aussi accepter de regarder en face la question: veut-on rendre son enfant aimable pour les autres? Le risque actuel est que le socius ne régule plus le fonctionnement narcissique indifférencié intrafamilial.

3) Le troisième niveau est la loi qui transmet des interdits reposant pour la plupart sur le principe: «ne fais pas à autrui ce que tu ne voudrais pas qu'on te fasse». Et lorsque la parole du magistrat ne suffit pas, ne fait plus tiers, alors la justice peut exercer une violence d'État; une sanction est envisagée sans idée de vengeance. L’inter-dit devient interdiction généralisée. Et une limite corporelle peut être nécessaire lorsque l'interdit verbal se révèle inopérant. Le sujet ne peut alors ressentir l'interdit qu'en faisant l'expérience d'une incapacité d'agir imposée par autrui. Le but est que le sujet éprouve une sensation et un sentiment de contenance, reprise à un niveau intrapsychique ou psychosomatique d'une expérience qui entrave 
une action violente (Berger, 2016). Ce terme de contenance est peu compris par les personnes qui ne raisonnent que selon l'opposition idéologique «éducatif versus répressif».

\section{Que se passe-t-il dans notre pratique clinique?}

L'interdit vient questionner toutes les zones de fragilité du psychisme parental.

1) Dans certaines situations familiales, il n'y a pas d'interdit, ou en tout cas pas d'interdit cohérent. Comme l'indique Tilmans (conversation personnelle), il est alors important de faire la différence entre analyse de la demande et analyse de la plainte. Ainsi il arrive que ce soit l'école qui demande le rendez-vous chez un psychologue ou pédopsychiatre, alors que les parents ne se plaignent pas du comportement de leur enfant et trouvent qu'il n'y a pas de problème. Dans un tel contexte, on a difficilement d'autre choix que d'attendre que la situation empire. Sinon, les parents acceptent des soins uniquement pour montrer qu'ils sont de bons parents qui font tout ce qu'on leur dit de faire pour leur enfant, mais on va presque toujours vers un échec. Donc vouloir "gagner du temps» parce qu’on a perçu une certaine souffrance chez l'enfant en fait perdre en réalité. On peut au mieux tenter un abord familial comme celui décrit ci-dessous, mais un abord individuel a de fortes chances d'échouer.

2) Certains parents présentent un Surmoi excessif, trop sévère appelé «sur-anti-moi» (Racamier), qui correspond parfois à ce que Freud a nommé «névrose de caractère»: "Je suis fait comme ça (au lieu de "on m’a fait comme ça”), et c'est-à-vous de vous adapter à moi». S'opposer aux interdits émis par un tel parent serait risqué de la part d'un enfant. Et si, une fois devenu adulte, il parvient à dire calmement à ce parent qu'il pense différemment de lui dans certaines circonstances, ce dernier risque de s'effondrer avec le sentiment que tout échappe à son contrôle.

3) Certains groupes familiaux présentent un fonctionnement clanique qui exige une similitude totale de pensée et qui interdit toute possibilité de différenciation. C'est pourquoi un homme maghrébin de 27 ans qui a une compagne depuis trois ans n'ose toujours pas le dire à son père qui exige de choisir les futurs conjoints de ses enfants. De tels systèmes comprennent de $l^{\prime}$ «incestuel» et du «meurtriel» (Caillot, 2016). «Incestuel» au sens où il 
faut se soumettre complètement au désir du parent, y compris dans le choix du partenaire sexuel - c'est le cas des filles soumises à un mariage arrangé-, et «meurtriel» au sens où un sujet qui se positionne de manière différente est exclu de la filiation, n'existe plus aux yeux des autres. Dans le fonctionnement clanique, l'extérieur est mauvais, l'intérieur est indifférencié, incritiquable, même s'il s'y produit des meurtres.

4) Le fonctionnement transgressif concerne des parents qui investissent la transgression. À la différence de certains parents qui pensent que la loi, c'est eux, et que la filiation se résume à une possession, ce qui intéresse les parents pervers transgressifs, c'est l'existence de la loi et la jouissance qu'ils éprouvent à la contourner. Ainsi un père s'arrange pour donner son urine dans un laboratoire à la place de son fils âgé de 15 ans afin que la justice n'y trouve pas de trace de cannabis. Et la mère de cet adolescent, ignorant cet échange, est tellement heureuse du résultat négatif de l'analyse qu'elle achète à son fils un habit de luxe pour le remercier d'avoir arrêté de se droguer. Un tel parent séduit son enfant en lui proposant une complicité malsaine dans le sabotage de toutes les formes d'autorité, dont celle du magistrat qu'il peut parvenir, avec ruse, à convaincre de ne pas prendre des mesures indispensables.

\section{Comment aborder les difficultés de fonctionnement familial concernant les interdits et leurs conséquences individuelles?}

Quelques remarques peuvent aider.

- Tout d'abord, abandonner ses propres normes éducatives. René Roussillon parle d'«hygiène psychique» du thérapeute, qui consiste à suspendre provisoirement son jugement de valeur sur les attitudes éducatives parentales, sauf s'il est nécessaire d'en prévenir rapidement des conséquences graves (Berger, 2012).

- Tenter de comprendre la logique éducative des parents. Freud disait qu'en pays névrotique, on utilise la monnaie névrotique (ou psychotique ou autre). Pourquoi ces parents pensent-ils que leur attitude éducative est la meilleure pour leur enfant? Même si les principes éducatifs des parents nous semblent nocifs à juste titre, il est important de faire preuve alors d'une curiosité naïve, d'une mentalité de chercheur: qu'est-ce que cette famille va m'apprendre? On peut faire remarquer aux parents que, dans d'autres familles, on aborde de telle ou telle manière le problème éducatif qu'ils évoquent. Pourquoi eux ont-ils préféré choisir cette solution? 
- Ne jamais accepter telle quelle la phrase parentale «Je sais que je fais mal» et demander alors "Qui vous a dit cela? Françoise Dolto, le mari, la belle-mère?» Il apparaît alors souvent que cette phrase est dite en faux-self, car si un parent agit ainsi, c'est qu'il est convaincu au fond de lui que c'est mieux pour son enfant. Et lorsqu'on explore l'histoire de ce parent, on va fréquemment trouver une dépression liée à des difficultés de relation avec ses propres parents. On peut simplement souligner que dans l'esprit du parent existent deux parties, deux manières de penser qui sont en désaccord.

- Explorer les scènes prototypiques de la vie familiale, c'est-à-dire celles où la question de l'espace partagé, des moments partagés se posent. Ce sont essentiellement:

- le moment du coucher, prototype de la séparation, où chacun part dans son espace. Comment chacun, enfant ou adulte, supporte-t-il cela?;

- les moments d'intimité corporelle: la salle de bain est-elle fermée lors de la douche d'un parent? L'enfant essaye-t-il d'entrer malgré l'interdit? Idem pour les WC;

- le moment où un parent demande l'arrêt des jeux vidéo ou d'internet;

- le moment du départ le matin, lorsque le parent doit aller travailler et l'enfant doit aller à l'école (obligations sociales);

- le moment du repas commun.

Au cours de cette exploration, il est nécessaire de ne jamais se contenter d'un terme général - «agressif», "opposant», etc. -, mais de faire un zoom précis sur comment s'est déroulée la scène: qu'avez-vous dit ou fait; qu'est-ce que votre enfant a alors dit ou fait; qu'avez-vous répondu; de quoi auriez-vous eu peur si vous aviez exigé telle chose ou, au contraire, autorisé telle autre? On peut consacrer deux séances d'une heure à déployer une séquence familiale qui a duré quelques minutes. Le but est de transformer des faits en événements, c'est-à-dire d'accéder aux affects qui étaient présents alors chez chacun, en particulier chez les parents.

Ceci permet de demander aux parents comment ces problèmes étaient envisagés dans leurs familles lorsqu'ils étaient enfants: pouvaient-ils dire non; ressentaient-ils les limites comme injustes, excessives; ou se sont-ils sentis en danger parce qu'il n'y avait pas de limites suffisamment protectrices; etc.? 
1) Avec cette exploration - «il était une fois une histoire familiale»débute la recherche des projections dont l'enfant a pu faire l'objet dès sa naissance. L'exemple suivant montre comment un parent peut se trouver soumis à un tiraillement identificatoire insupportable dès la naissance de son enfant. Un père envisage de donner une fessée à son fils âgé de deux jours parce qu'il ne prend pas bien le sein, difficulté courante lors d'un début d'allaitement. Pour lui, ce bébé est un "gâcheur». Interrogé sur ce mot, il explique que son père exigeait de ses enfants qu'à la fin de chaque repas, ils ramassent toutes les miettes restant sur la table afin de ne rien gâcher. Donc, soit il continue à s'identifier à son père, avec un risque important de maltraitance; soit il commet un «meurtre éducatif» de son père, en élevant son enfant avec tolérance et empathie, mais alors apparaîtra en lui la douleur de penser à la manière dont son père a gâché son enfance et l'a empêché de ressentir l'insouciance normale de tout enfant.

On tente donc ainsi de faire évoquer aux parents un tiers interne, c'està-dire l'enfant en eux, et l'expérience négative de l'interdit qu'ils ont pu faire et qui les empêche d'en voir la valeur pour leur enfant. Mais de nos jours, avec le lâchage des interdits au niveau du groupe social, ce travail est devenu beaucoup plus difficile à réaliser, et le thérapeute est parfois amené à prendre une position de tiers dans la réalité, en se prescrivant lui-même une ordonnance indiquant les règles de vie commune à la maison, écrit auquel les parents se réfèrent lorsqu'existe un conflit d'autorité avec leur enfant. Ceci ne peut être conçu que comme un retour en arrière par un psychanalyste, mais c'est parfois incontournable.

2) Une autre possibilité consiste à se projeter dans le futur, c'est-à-dire à demander aux parents comment ils imaginent la situation quinze ans plus tard. Ainsi, cette mère et son adolescent fortement délinquant et violent vivent dans une bulle avec le fantasme qu'ils doivent se protéger l'un l'autre. L'enfant protège la mère de la grave dépression qu'elle a traversée pendant plus de quatre ans lorsque son mari est mort alors qu'il avait quinze mois. Et la mère a alors décidé de protéger son enfant car elle considère qu'il a tout de suite ressenti qu'il était en grave manque de père. Point fondamental, on constate ici comment dans beaucoup de familles décrites comme fusionnelles, avec des relations parent-enfant dites érotisées, l'organisation familiale est avant tout une défense contre un fantasme de catastrophe. Je demande alors comment cette mère imagine la situation dans dix ans. Sa réponse est que son fils sera marié, qu'il aura des enfants et un travail. Et comment vivront-ils? Comme elle a un appartement de quatre pièces, 
elle pense que son fils et sa femme viendront vivre chez elle ou qu'elle ira vivre chez eux. Le fils m'a dit exactement la même chose lorsque je l'ai reçu seul auparavant. À chacun, reçu séparément, je pose la question de ce qui se passera si la femme du fils a envie d'avoir un espace personnel pour le couple et ne souhaite pas avoir sa belle-mère au domicile. Le fils répond que sa femme devra "dégager», alors que la mère est plus nuancée, répondant qu'elle ira vivre la moitié du temps dans sa famille en Algérie, où elle se sent heureuse, et qu'elle pourra rester seule dans un studio le reste du temps. Mais les paroles des uns et des autres nécessitent d'être prononcées lors d'un entretien commun, afin d'introduire un tiers, ce que le garçon refuse pour le moment.

Pour terminer, j'ajouterai qu'on peut avoir l'illusion que ces situations sont simples, qu'il s'agit seulement d'un problème "éducatif», que les parents ont l'air d'accord avec nous sur le bien-fondé de l'attitude la meilleure à adopter avec leur enfant. En réalité, obtenir des changements est souvent difficile, voire aléatoire. D'autant plus que, lorsque les parents évoluent, l'enfant, lui, n'a parfois aucune intention de renoncer à ses avantages acquis.

Maurice Berger drmberger@laposte.net

\section{Notes}

1. Cet article rappelle un des dix commandements: "Tu honoreras ton père et ta mère». Cependant un parent n'est respectable qu'autant qu'il respecte son enfant, plus faible et plus vulnérable que lui.

\section{Références}

Berger, M. (2012). Le travail thérapeutique avec la famille. Paris: Dunod.

Berger, M. (2016). De l'incivilité au terrorisme. Comprendre la violence sans l'excuser. Paris: Dunod.

Bion, W. (1959). Recherches sur les petits groupes. Paris: Presses universitaires de France, 1965. Caillot, J. P. (2016). Le meurtriel, l'incestuel et le traumatique. Paris: Dunod.

Carel, A. (2002). Le processus d'autorité. Revue française de psychanalyse, 66 (1), 21-40.

Carel, A. (2016). Croire au lien entre autorité et bien-être! Communication présentée au colloque «Le bien-être et le processus d'autorité», dans le cadre du Congrès annuel du collège de psychanalyse groupale et familiale, Paris. Document inédit.

Ciccone, A. (2016). La parentalité soignante. Dans Violences dans la parentalité (p. 9-28). Paris: Dunod.

Grunberger, B. (2003). Le narcissisme. Paris: Payot Rivages.

Lacroix, M. B. (1999). Nous sommes tous des volés voleurs. Dans Enfants terribles, enfants féroces (p. 159-160). Paris: Éditions Érès.

Racamier, P.-C. (2016). Le deuil originaire. Paris: Payot. 
26 Filigrane, 2017

Roussillon, R. (2014). Manuel de psychologie clinique et psychopathologie. Paris: Elsevier Masson.

Winnicott, D. W. (1963). Élaboration de la capacité de sollicitude. Dans Processus de maturation chez l'enfant (p. 31-42). Paris: Payot, 1970. 